\title{
Influência da reabilitação pulmonar sobre o padrão de sono de pacientes portadores de doença pulmonar obstrutiva crônica*
}

\author{
Influence of pulmonary rehabilitation on the sleep patterns of patients with \\ chronic obstructive pulmonary disease \\ RENATA CLAUDIA ZANCHET*, CARLOS ALBERTO DE ASSIS VIEGAS**(TE SBPT) \\ TEREZINHA DO SOCORRO MACÊDO LIMA**(TE SBPT)
}

Introdução: A Reabilitação Pulmonar (RP) melhora a qualidade de vida de pacientes portadores de doença pulmonar obstrutiva crônica (DPOC). No entanto, o papel da RP sobre o padrão de sono destes pacientes ainda não está estabelecido.

Objetivo: Avaliar a influência da RP sobre o padrão de sono de pacientes portadores de DPOC.

Método: Foram estudados 27 pacientes (22 homens), que foram submetidos a exames espirométricos, gasométricos, antropométricos e polissonográficos antes e depois de seis semanas de RP, além de responderem a escala de sonolência de Epworth. A análise estatística foi realizada pelo teste t de Student para amostras pareadas, ANOVA e o teste de comparações múltiplas Newmans-Keuls.

Resultados: Observamos que os pacientes estudados tinham idade média de $63 \pm 5$ anos, VEF $=55 \pm 25 \%$ do previsto, $\mathrm{VEF}_{1} / \mathrm{CVF}=50 \pm 12 \%, \mathrm{PaO}_{2}$ em repouso de $70 \pm 7 \mathrm{mmHg}$ e $\mathrm{SaO}_{2}$ igual a $94 \pm 2 \%$. A polissonografia revelou sono fragmentado, redução do sono delta e dessaturação da hemoglobina, cujas maiores quedas ocorreram durante o sono REM. Não houve diferença estatisticamente significativa $(p>0,05)$ na comparação entre as variáveis estudadas antes e após RP.

Conclusão: No grupo de pacientes estudados, o programa de RP não modificou o padrão de sono.
Background: Pulmonary Rehabilitation (PR) improves the quality of life of chronic obstructive pulmonary disease (COPD) patients. However, the influence of PR on the sleep pattern of these patients is unknown.

Objective: To evaluate the influence of PR on the sleep patterns of patients with COPD.

Method: A total of 27 patients (22 men/5 women) were submitted to polysomnographic, gasometric and anthropometric studies before and after six weeks of PR and were evaluated using the Epworth Sleepiness Scale. The results were analyzed using paired Student's $t$-test, ANOVA and Newman-Keuls multiple comparison test.

Results: Mean age was $63.3 \pm 5.3$ years, mean $\mathrm{FEV}_{1}$ was $54.8 \pm 25.4 \%$ of predicted, mean $\mathrm{FEV}_{1} / \mathrm{FVC}$ was $49.9 \pm 12.0 \%$ of predicted, mean resting $\mathrm{PaO}_{2}$ was 69.7 $\pm 7.3 \mathrm{mmHg}$, and mean resting $\mathrm{SaO}_{2}$ was $93.7 \pm 2.1 \%$. Polysomnography revealed sleep patterns to be fragmented, with frequent waking and reduced slowwave sleep, as well as oxygen desaturation. The most significant drops in oxygen saturation occurred during rapid eye movement sleep. No significant differences were observed between pre- and post-PR values for the other variables studied $(p>0.05)$.

Conclusion: In the group of patients studied, PR did not alter sleep patterns.

J Bras Pneumol 2004; 30(5) 439-44

Descritores: Sono. DPOC. Reabilitação Pulmonar.

Key words: Sleep. Pulmonary Disease. Chronic Obstructive. Lung Diseases, rehabilitation.

* Trabalho realizado na Universidade Católica de Brasilia (UCB) e **Hospital Universitário de Brasília (HUB), Brasilia, DF Endereço para correspondência: Renata Claudia Zanchet. Universidade Católica de Brasília. Curso de Fisioterapia. QS 07, Iote 01, Águas Claras. CEP: 71966-700 Taguatinga - DF. Brasil

Tel: 55-61-356 9205 - Fax: 55-61-356 1800 - E-mail: renatac@ucb.br

Recebido para publicação, em 4/11/03. Aprovado, após revisão em 29/4/04. 


\section{INTRODUÇÃO}

A atividade física, a depender de sua modalidade, duração, horário de realização e freqüência pode influenciar o sono de indivíduos saudáveis $s^{(1,2)}$. Em estudo de meta-análise ${ }^{(1)}$, foi demonstrado que exercícios físicos realizados a curto e longo prazo aumentam o sono delta (estágios 3 e 4), o tempo total de sono (TTS) e diminuem as latências de sono e do sono REM.

King e colaboradores ${ }^{(3)}$ avaliaram 0 ef eito de 16 semanas de exercícios físicos de moderada intensidade em 67 indivíduos com faixa etária entre 50 e 76 anos, sedentários, sem diagnóstico médico de distúrbios do sono, porém que queixavam de despertar durante a noite e/ou apresentar dificuldades para iniciar o sono. Os autores demonstraram que, após o referido programa de atividade física, os indivíduos melhoraram a qualidade do sono. Além disto, verifica-se que, apesar de existir correlação entre a saturação periférica de oxigênio $\left(\mathrm{SpO}_{2}\right)$ durante o sono e o exercício ${ }^{(7,8)}$, a queda na saturação durante o exercício não é boa preditora da dessaturação noturna, uma vez que pequena queda na $\mathrm{SpO}_{2}$ durante a atividade física não exclui maiores índices de diminuição na saturação durante a noite ${ }^{(7,9)}$.

Por outro lado, em pacientes portadores de doença pulmonar obstrutiva crônica (DPOC), foi demonstrado que episódios de hipoxemia e hipercapnia noturnas estão intimamente relacionados aos períodos de sono REM $^{(4,5)}$, de tal forma que os mais baixos níveis noturnos de $\mathrm{SpO}_{2}$ foram encontrados em pacientes que tinham hipoxemia diurna mais grave e períodos mais longos de sono REM ${ }^{(6)}$.

Além de alterações gasométricas, pacientes portadores de DPOC apresentam outras alterações na qualidade do sono ${ }^{(10,11)}$. Como este tende a ser fragmentado, com despertares freqüentes ${ }^{(12,13)}$, há diminuição dos sonos delta e REM ${ }^{(12)}$, do tempo total de sono e aumento das mudanças de estágio ${ }^{(14,15)}$. Apesar disto, os distúrbios do sono são freqüentemente ignorados pela maioria dos médicos na avaliação de pacientes portadores de DPOC, mesmo em protocolos de pesquisas desenhados para avaliar a qualidade de vida destes pacientes ${ }^{(12)}$.

Embora a literatura apresente várias pesquisas sobre o sono em pacientes portadores de DPOC, a relação entre a Reabilitação Pulmonar (RP) e o sono destes pacientes ainda não foi estudada. Portanto, 0 objetivo deste trabalho foi verificar a influência da RP sobre 0 padrão de sono de pacientes portadores de DPOC.

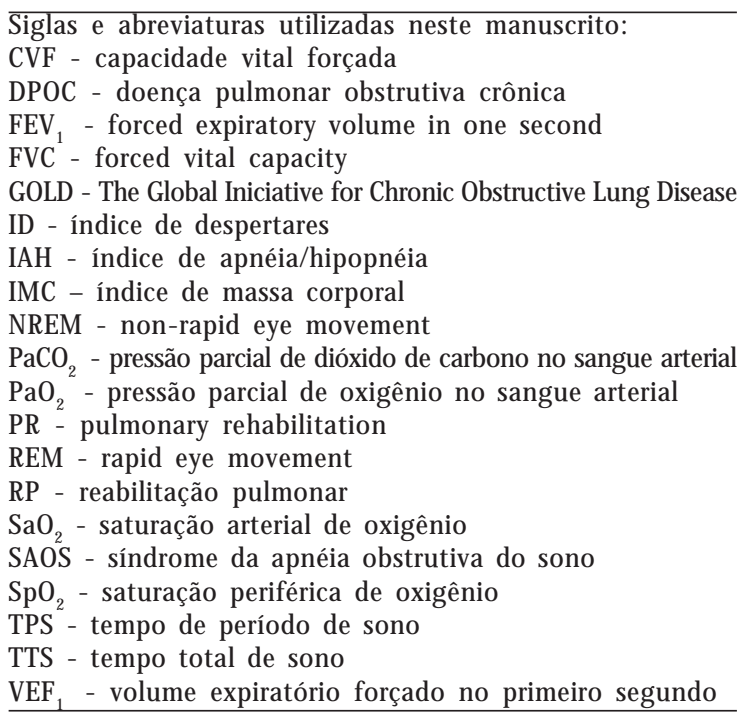

\section{MÉTODO}

Foi realizado um ensaio clínico não randomizado e aberto, no qual foram avaliados 35 pacientes portadores de DPOC. Destes, oito foram excluídos: três por apresentarem síndrome da apnéia obstrutiva do sono (SAOS), dois por se recusarem a repetir a polissonografia depois da RP, um por desenvolver problema ortopédico durante as sessões de treinamento, um por apresentar grave arritmia durante o programa e outro por hipertensão arterial descompensada. Portanto, o estudo foi realizado com 27 pacientes, sendo 22 do sexo masculino, no período de agosto de 2001 a abril de 2003. A DPOC foi diagnosticada de acordo com critérios definidos pelo The Global Iniciative for Chronic Obstructive Lung Disease ${ }^{(16)}$. A SAOS foi caracterizada por um índice de apnéia hipopnéia por hora de sono (IAH) superior a cinco ${ }^{(17)}$.

Neste estudo foram incluídos pacientes portadores de DPOC estáveis clinicamente e ex-tabagistas há, no mínimo, três meses. Foram excluídos pacientes com SAOS, com doença cardiovascular ou ortopédica que os impossibilitassem de realizar os exercícios do protocolo de RP, ou que apresentassem outra comorbidade que os colocassem em risco durante a realização dos exercícios.

0 trabalho foi aprovado pelo Comitê de Ética do Hospital Universitário de Brasília. Todos os pacientes leram e assinaram o termo de consentimento informado. 
Para avaliar o grau de obstrução e o estado clínico dos pacientes foram realizados, em dois momentos, exames espirométricos, gasométricos, antropométricos, polissonográficos e aplicada a escala de sonolência de Epworth. O primeiro momento de avaliação foi antes da RP e o outro imediatamente após a RP.

Função pulmonar: Foram medidos os valores absolutos da capacidade vital forçada (CVF), do volume expiratório forçado no primeiro segundo $\left(V F_{1}\right)$ e da relação $V F_{1} / C V F \%$ (Vmax-22 series spirometer Sensor M edics Yorba Linda, Califórnia, USA) e calculados os valores relativos aos previstos para o sexo, a idade e a altura, considerando os valores descritos por Knudson e colaboradores ${ }^{(18)}$. Para a realização da espirometria seguiram-se as normas da American Thoracic Society ${ }^{(19)}$.

Gasometria arterial: Foram medidos os valores da pressão parcial de oxigênio no sangue arterial $\left(\mathrm{PaO}_{2}\right)$, pressão parcial de dióxido de carbono no sangue arterial $\left(\mathrm{PaCO}_{2}\right)$, e $\mathrm{SaO}_{2}$ (saturação arterial de oxigênio) (Ciba Corning 278 Gas System, Ciba Corning, Diagnostics Corp, M edifield, USA).

Antropometria: Foram avaliados o índice de massa corporal (IMC), calculado pela fórmula: peso I altura ${ }^{2}\left(\mathrm{Kg} / \mathrm{m}^{2}\right)$ e a circunferência do pescoço (cm), mensurada acima da cartilagem cricóide.

Polissonografia de noite inteira: Os parâmetros monitorados foram eletroencefalograma, eletrocardiograma, eletromiograma, eletrooculograma, fluxo aéreo nasal/bucal, posição corporal, ronco, movimento torácico e abdominal, além da saturação periférica de oxigênio $\left(\mathrm{SpO}_{2}\right)$. Estas mensurações foram realizadas por aparelho da marca Healthdyne technologies, tipo Alice $3^{\circ}$ computadorized Polyssonographic System. Foram avaliadas as variáveis habituais da polissonografia, de acordo com Rechtschaffen e Kales ${ }^{(20)}$. Incluímos, também, o índice de despertares(ID), que é o resultado da relação entre o número de despertares/ tempo de período de sono, multiplicado por 60 , indicando o número de despertares por hora de sono.

Escala de Sonolência de Epworth: A escala de sonolência criada por Johns ${ }^{(21)}$ foi respondida por todos os pacientes antes e depois do programa de RP.

Treinamento físico: 0 programa de RP durou seis semanas, com freqüência de três vezes semanais, sempre pela manhã. A sessão de treinamento físico do programa de RP seguiu as normas descritas por Rodrigues e colaboradores ${ }^{(22)}$. 0 efeito da RP foi avaliado pelo teste de caminhada de seis minutos e pelo teste incremental de membros superiores.

Estatística: As variáveis estudadas são apresentadas em seus valores médios \pm desvio padrão (DP). Para comparação entre as mesmas antes e após o programa de RP, foi utilizado 0 teste $t$ de Student para amostras pareadas. Para comparar a diferença entre os níveis de $\mathrm{SpO}_{2}$ em vigília noturna, durante o sono NREM e REM utilizou-se ANOVA e o teste de comparações múltiplas Newmans-Keuls. Consideramos diferenças estatisticamente significativas quando $p<0,05$.

\section{RESULTADOS}

A média de idade dos pacientes estudados foi de 63,3 $\pm 5,3$ anos, variando de 54 a 72 anos.

$\mathrm{Na}$ tabela 1 apresentamos os valores das variáveis espirométricas, gasométricas e antropométricas avaliadas antes e após programa de RP e, conforme podemos observar, não houve diferença estatisticamente significativa entre estas variáveis $(p>0,05)$.

TABELA 1

Valores da espirometria, gasometria, antropometria e escala de Epworth dos pacientes portadores de DPOC estudados antes e após programa de RP.

\begin{tabular}{lrr}
\hline & Antes da RP & Após RP \\
\hline CVF (\%) & $85,0 \pm 26,6$ & $87,4 \pm 22,5$ \\
VEF $_{1}(\%)$ & $54,8 \pm 25,4$ & $55,0 \pm 22,5$ \\
$\operatorname{VEF}_{1} /$ CVF (\%) & $49,9 \pm 12,0$ & $49,5 \pm 12,4$ \\
$\mathrm{PaO}_{2}(\mathrm{mmHg})$ & $69,7 \pm 7,3$ & $71 \pm 8,7$ \\
$\mathrm{PaCO}_{2}(\mathrm{mmHg})$ & $35,0 \pm 4,8$ & $34,7 \pm 4,6$ \\
$\mathrm{SpO}_{2}$ diurna (\%) & $93,7 \pm 2,1$ & $93,7 \pm 2,2$ \\
$\mathrm{IMC}^{\mathrm{M}}\left(\mathrm{Kg} / \mathrm{m}^{2}\right)$ & $24,6 \pm 4,2$ & $24,6 \pm 4,1$ \\
Circunferência do pescoço (cm) & $37,5 \pm 3,7$ & $37,9 \pm 3,7$ \\
Escala de Epworth & $9 \pm 4$ & $9 \pm 4$ \\
\hline
\end{tabular}


O exame polissonográfico revelou sono fragmentado, diminuição do sono delta e dessaturação da hemoglobina. A $\mathrm{SpO}_{2}$ antes da RP durante 0 sono REM $(88 \pm 4,2 \%)$ foi significativamente menor que durante a vigília noturna $(90 \pm 2,9 \%)$ e o sono NREM $(89,4 \pm 3,3 \%)(p<0,05)$. Da mesma forma, a $\mathrm{SpO}_{2}$ no sono NREM foi menor que em vigília noturna $(p<0,05)$ (Figura 1$)$.

Não houve diferença estatisticamente significativa entre as variáveis polissonográficas e o nível de sonolência diurna medidos antes e após RP ( $p>0,05)$ (Tabela 2).

Quanto à RP, houve um aumento significativo na distância percorrida no teste de caminhada de seis minutos e na carga máxima atingida no teste incremental de membros superiores $(p<0,05)$.

\section{DISCUSSÃO}

Com o presente estudo foi possível caracterizar o padrão do sono de pacientes portadores de DPOC. Quando comparamoso sono dos pacientes estudados neste trabalho com o padrão do sono de indivíduos adultos saudáveis ${ }^{(23)}$, verificamos que os portadores de DPOC apresentavam sono fragmentado, caracterizado por aumento do tempo de vigília durante o período total de sono e pelo alto índice de despertares. Observamos, também, baixa eficiência do

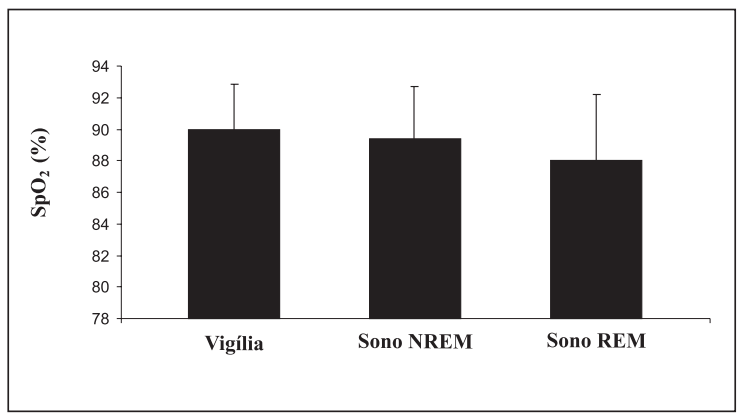

Figura 1 - Comparação entre valores da $\mathrm{SpO}_{2}$ em vigília noturna e durante o sono

sono, além da dificuldade em iniciar o sono (aumento das latências de sono e do sono REM) e em aprofundálo (aumento da porcentagem do estágio 1 do sono NREM e diminuição da porcentagem do sono delta). Estes dados estão de acordo com os resultados encontrados por outros autores ${ }^{(12-15)}$.

0 al to índice de despertares e a dificuldade em iniciar o sono são corroborados por George $\mathrm{e}^{(24)}$ que, em revisão de literatura, chama a atenção para a presença de insônia nos portadores de DPOC.

Alguns pacientes portadores de DPOC apresentam, concomitantemente, a síndrome da apnéia obstrutiva

TABELA 2

Valores polissonográficos dos pacientes portadores de DPOC estudados antes e após programa de RP.

\begin{tabular}{|c|c|c|}
\hline & Antes da RP & Após RP \\
\hline IAH (eventos/hora) & $2,3 \pm 3,9$ & $2,8 \pm 5,2$ \\
\hline Tempo de ronco (minutos) & $10,9 \pm 9,1$ & $9,8 \pm 7,3$ \\
\hline Tempo de registro (minutos) & $486,4 \pm 46,0$ & $483,2 \pm 32,4$ \\
\hline Tempo de período de sono - TPS (minutos) & $441,9 \pm 67,1$ & $442,7 \pm 39,1$ \\
\hline Tempo total de sono - TTS (minutos) & $321,3 \pm 75,8$ & $323,1 \pm 69,1$ \\
\hline TTS / TPS (\%) & $72,8 \pm 13,9$ & $72,7 \pm 12,7$ \\
\hline \% vigília durante o TPS (\%) & $27,2 \pm 13,9$ & $27,3 \pm 12,7$ \\
\hline Latência do sono (minutos) & $28,8 \pm 28,5$ & $27,3 \pm 23,1$ \\
\hline Latência do sono REM (minutos) & $113,9 \pm 73,0$ & $105,6 \pm 56,3$ \\
\hline Mudanças de estágios & $126,8 \pm 35,2$ & $117,1 \pm 33,6$ \\
\hline Episódios de movimentos & $40,1 \pm 14,6$ & $37,2 \pm 14,3$ \\
\hline Índice de despertares (eventos/hora) & $30,3 \pm 10,0$ & $30,9 \pm 8,2$ \\
\hline Estágio $1(\%)$ & $20,7 \pm 7,6$ & $19,7 \pm 8,8$ \\
\hline Estágio 2 (\%) & $49,0 \pm 8,3$ & $48,7 \pm 9,5$ \\
\hline Sono delta (\%) & $8,8 \pm 4,9$ & $9,5 \pm 7,4$ \\
\hline Sono REM (\%) & $19,9 \pm 6,4$ & $20,6 \pm 7,0$ \\
\hline Tempo de $\mathrm{SpO}_{2}<90 \%(\%)$ & $40,1 \pm 37,8$ & $45,1 \pm 37,2$ \\
\hline $\mathrm{SpO}_{2}(\%)$ - média em vigília & $90,0 \pm 2,9$ & $90,6 \pm 2,0$ \\
\hline $\mathrm{SpO}_{2}(\%)$ - média no NREM & $89,4 \pm 3,3$ & $89,7 \pm 2,2$ \\
\hline $\mathrm{SpO}_{2}^{2}(\%)$ - média no REM & $88,0 \pm 4,2$ & $87,5 \pm 3,6$ \\
\hline
\end{tabular}


do sono (SAOS). A coexistência da DPOC com SAOS, designada overlap syndrome, pode agravar a hipoxemia noturna destes pacientes ${ }^{(10,11)}$. Em nosso estudo, esta síndrome esteve presente em 8,6\% da população avaliada. Este valor está próximo daqueles apresentados por Chaouat e colaboradores ${ }^{(25)}$, que encontraram a SAOS em $11 \%$ dos 265 pacientes estudados. É difícil avaliar a real associação entre DPOC e SAOS devido, principalmente, a dois fatores. Em algumas pesquisas, ocorre falha na triagem do paciente, como no estudo de Guilleminault e colaboradores ${ }^{(26)}$, que avaliaram pacientes portadores de DPOC cuja maioria havia sido encaminhada à clínica de sono por apresentar hipersonolência diurna. Estes pesquisadores encontraram a overlap syndrome em $84 \%$ dos pacientes. Outro fator é a falta de consenso, entre os diferentes autores ${ }^{(24,27-30)}$, sobre 0 número de episódios de apnéia e hipopnéia que caracteriza a SAOS.

Douglas ${ }^{(11)}$ e McNicholas(31), em revisão bibliográfica, definem alguns fatores que podem ser responsáveis pela hipoxemia durante o sono dos pacientes portadores de DPOC. Estes fatores incluem a hipoventilação alveolar, a diminuição da capacidade residual funcional, além da alteração na relação ventilação/perfusão. Os pacientes hipoxêmicos têm maiores índices de dessaturação perante à hipoventilação quando comparados com os pacientes normoxêmicos, pelo efeito da curva de dissociação da oxihemoglobina(31).

0 presente estudo confirma os dados apresentados na literatura de que ocorre queda na $\mathrm{SpO}_{2}$ durante o sono e que o maior nível de dessaturação nos portadores de DPOC ocorre durante o sono REM ${ }^{(14,27)}$.

Atendendo aos objetivos deste trabalho, apesar da efetividade sobre a capacidade de exercício, verificamos que seis semanas de RP não alteraram os dados obtidos na polissonografia dos 27 pacientes portadores de DPOC estudados. Portanto, o resultado encontrado em nosso trabalho mostra que a resposta do portador de DPOC ao exercício pode ser diferente dos indivíduos normais, que apresentam melhora da qualidade do sono após a realização de treinamento físico ${ }^{(1,3)}$.

Kubitz e colaboradores ${ }^{(1)}$, em trabalho de meta análise, constataram que tanto o exercício agudo (isto é, exercício x não-exercício) quanto o crônico (isto é, treinados $x$ não-treinados; atletas $x$ não atletas) promovem aumento do sono delta e do tempo total de sono, além de diminuir a latência do sono e do sono REM. Porém, o ef eito do exercício crônico sobre o sono é maior que do agudo. Além disto, o impacto no sono é maior quando o exercício é aeróbio, de longa duração e realizado em horários não muito próximos à hora de dormir. Os trabalhos utilizados nesta meta análise referem-se a exercícios realizados entre 14:00h e 19:00h e a duração dos exercícios geralmente era em torno de uma hora.

Segundo Horne e Staff ${ }^{(32)}$, pode ocorrer aumento do sono de ondas lentas sempre que houver aumento da temperatura corporal durante o exercício, mas esta hipótese é muito questionada, pois a temperatura corporal retorna ao valor basal em torno de duas horas após o exercício ${ }^{(33)}$ e, Youngstedt e coloboradores ${ }^{(2)}$ concluíram que a temperatura não modera o sono delta após o exercício.

Um dos fatores que pode ter contribuído para que a RP não tenha alterado o sono dos nossos pacientes foi o período de treinamento muito curto (seis semanas) e a duração de trinta minutos por sessão, tempo que também está abaixo da maioria dos trabalhos realizados com indivíduos sem doença pulmonar. Contudo, acreditamos que 0 principal determinante para melhorar o sono nestes indivíduos após a realização de exercícios é o período do dia em que é executado. Esta hipótese é corroborada por Driver e Taylor ${ }^{(34)}$, os quais afirmam que o exercício realizado pela manhã, independente da intensidade ou duração, provavelmente não altera 0 padrão de sono de indivíduos saudáveis, diferentemente do exercício realizado no final da tarde. Além disto, todos os estudos utilizados na meta análise de Kubitz e colaboradores ${ }^{(1)}$ relatam a realização de exercícios à tarde ou à noite.

Contudo, quando se refere a pneumopatas crônicos, além de todos estes fatores que podem influenciar 0 sono, temos 0 fato de que estes pacientes podem ser hipoxêmicos crônicos. Como citado anteriormente, a hipoxemia leva à fragmentação do sono. Uma vez que esta condição não se altera após a RP, o padrão de sono deve manter-se inalterado.

Pelo exposto, entendemos que mais pesquisas devam ser realizadas, incluindo um período mais prolongado de RP, sendo esta executada em períodos diferentes do dia, para efeito de comparação.

Em resumo, podemos concluir que a RP não altera o padrão de sono de pacientes portadores de DPOC. 


\section{REFERÊNCIAS}

1. Kubitz KA, Landers DM, Petruzzello SJ, Han M. The effects of acute and chronic exercise on sleep. A metaanalytic review. Sports Med 1996;21:277-91.

2. Yongstedt SD, O'Connor PJ, Dishman RK. The effects of acute exercise on sleep: a quantitative syntesis. Sleep 1997;20:103-14.

3. King AC, Oman RF, Brassington GS, Bliwise DL, Haskell WL. Moderate-intensity exercise and self-rated quality of sleep in older adults: a randomized controlled trial. J AMA 1997;277:32-7.

4. McKeon J L, Muree-Allan K, Saunders NA. Prediction of oxygenation during sleep in patients with severe chronic obstructive lung. Thorax 1988;43:312-7.

5. Connaughton JJ, Catterall JR, Elton RA. Do sleep studies contribute to the management of patients with severe chronic obstructive pulmonary disease? Am Rev Respir Dis 1988; 138:341-4.

6. Little AS, Elkholy MM, Chalmer GW. Predictors of nocturnal oxygen desaturation in patients with COPD. Resp Med 1999; 93:202-7.

7. Mulloy E, McNicholas WT. Ventilation and gas exchange during sleep and exercise in severe COPD. Chest 1996;109:387-94.

8. Mulloy E, Fitvtatrich M, Bourke S, O'Regan A, MC Nicholas WT. Oxigen dessaturation during sleep and exercise in patients with severe chronic obstruction pulmonary disease. Respir Med 1995;89:193-8.

9. Fletcher EC, Luckett RA, Miller T, Fletcher J G. Exercise hemodynamics and gas exchange in patients with chronic obstruction pulmonary disease, sleep desaturation, and a daytime $\mathrm{PaO}_{2}$ above $60 \mathrm{mmHg}$. Am Rev Respir Dis 1989; 140:1237-45.

10. Jokic R, Fitzpatrick MF. Obstructive lung disease and sleep. Med Clin North Am 1996;80:821-50.

11. Douglas NJ. Sleep in patients with chronic obstructive pulmonary disease. Clin Chest Med 1998;19:115-25.

12. Ping-Shin T, MCDonnell M, Spertus JA et al. A new selfadministered questionnaire to monitor health-related quality of life in patients with COPD. Chest 1997;112:614-22.

13. Collard P, Dury M, Delguste P, Genevieve A, Rodenstein DO. Movemet arousals and sleep-related disordered breathing in adults. Am J Respir Crit Care Med 1996; 154:454-9.

14. Fleetham J, West P, Mezon B, Conway W, Roth T, Kryger M. Sleep, arousals, and desaturation in chronic obstructive pulmonary disease: the effect of oxygen therapy. Am Rev Respir Dis 1982;126:429-33.

15. Santos CEVG, Viegas CAA. Padrão do sono em pacientes portadores de DPOC e correlação entre variáveis gasométricas, espirométricas e polissonográficas. J Pneumol 2003;29:69-74.

16. GOLD. Global Strategy for the Diagnosis, Management, and Prevention of Chronic Obstructive Pulmonary Disease. Am J Respir Crit Care Med 2001;163:1256-76.

17. American Academy of sleep medicine. Sleep-related brething disorders in adults: recommendations for syndrome definitions and measurements techniques in clinical research. Sleep 1999;22:667-89.
18. Knudson RJ, Lebowitz MD, Holbverg CJ. Changes in the normal expiration flow-volume curve with growth and aging. Am Rev Respir Dis $1983 ; 127: 725-34$.

19. American Thoracic Society. Standardization of spirometry - 1994 update. Am J Respir Crit Care Med 1995;152:1107-36.

20. Rechtschaffen $A$, Kales $A$. A manual of standardized terminology, thecniques and scoringsystem for sleep stages of human subjects. Washington: U.S. Government Printing Office, 1968. Publication no 204.

21. Johns MW. A new method for measuring daytime sleepness: the Epworth Sleepiness Scale. Sleep 1991;14:540-5.

22. Rodrigues SL, Viegas CAA, Lima T. Efetividade da reabilitação pulmonar como tratamento coadjuvante da doença pulmonar obstrutiva crônica. J Pneumol 2002;28: 65-70.

23. Guiot M. Polissonografia. In: Reimão R. Sono estudo abrangente. 2 o ed. São Paulo: Atheneu, 2 ed., 1996;92-8.

24. George, C.F.P. Perspective on the management of insomnia in patients with chronic respiratory disorders. Sleep 2000;23:S31-5.

25. Chaouat A, Wetzenblum E, Krieger J, Ifoundza T, Oswald M, Kessler R. Association of chronic obstructive pulmonary disease and sleep apnea syndrome. Am J Respir Crit Care Med 1995;151: 82-6.

26. Guilleminaut C, Cumminskey J, Motta J. Chronic obstructive airflow disease and sleep studies. Am J Respir Crit Care Med 1980;122:397-406.

27. Donner CF, Braghiroli A, Sacco C, Carone M. Non-apnoeic nocturnal $\mathrm{O}_{2}$ desaturations in COPD patients with daytime borderline hypoxaemia. Eur Respir J 1990;11:538S-48S.

28. Norman JF, Von Essen SG, Fuchs RH, McElliot $M$. Exercise training effect on obstructive sleep apnea syndrome. Sleep Res Online 2000;3:121-9.

29. Chaouat A, Wetzenblum E, Kessler R, Schott $R$, Charpentier C, Levi-Valensi $P$, et al. Outcome of COPD patients with mild daytime hypoxaemia with or without sleep-related oxygen desaturation. Eur Respir J 2001;17:848-55.

30. Mayos M, Plaza LH, Farré A, Mota Y, Snachis J. Efeto de la oxigenoterapia nocturna en el paciente con síndrome de apnea-hiponea del sueño y limitación crónica al flujo aéreo. Arch Bronconeumol 2001;37:65-8.

31. MCNicholas WT. Impact of sleep in COPD. Chest 2000;117:48S-53S.

32. Horne JA, Staff LHE. Exercise and sleep: body-heating effects. Sleep 1983;6:36-6.

33. Gore, C.J .; Withers, R.T. Effect of exercise intensity and duration on postexercise metabolism. J Appl Physiol 1990;68: 2362-8.

34. Driver HS, Taylor SR. Sleep disturbances and exercise. Sports Med 1996;21:1-6. 